Ophthalmologe $2017 \cdot 114: 96-97$ DOI 10.1007/s00347-016-0402-x Online publiziert: 7. Dezember 2016 c) Springer Medizin Verlag Berlin 2016

CrossMark

\section{R. R. Böhm}

Zentrum für Augenheilkunde, Klinik für Erkrankungen des vorderen Augenabschnittes, Universitätsklinikum Essen, Essen, Deutschland

\title{
Alterndes visuelles System im Kontext neurodegenerativer Erkrankungen
}

„Das Auge, welches man auch das Fenster der Seele nennt" (Leonardo da Vinci [1452-1519])

Eine alternde Gesellschaft, bedingt durch einen demografischen Wandel, führt zu epidemiologischen, sozioökonomischen und auch medizinischen Herausforderungen. Aus medizinischer Sicht ist die Zunahme von altersbedingten Erkrankungen von zunehmender Bedeutung.

In den letzten Jahren wurden morphologische und molekularbiologische Kriterien des physiologischen Alterns des visuellen Systems intensiv erforscht. Während des Alterungsprozesses kommt es $\mathrm{zu}$ einer multifaktoriell bedingten $\mathrm{Ab}$ nahme der visuellen Leistungsfähigkeit. Neben morphologischen Alterungsvorgängen der präretinalen optischen Strukturen, wie Linse, Hornhaut und Glaskörper, werden Veränderungen innerhalb der Retina, in der aufsteigenden Sehbahn und auch im visuellen Kortex diskutiert. Von Bedeutung ist eine Auseinandersetzung mit dem Alterungsprozess des gesamten „System Auge“, der einzelnen Bestandteile einschließlich der aufsteigender Sehbahn und des visuellen Kortex. Durch den systemhaften Aufbau des Auges können sich Veränderungen einzelner Bestandteile auch auf Funktion und Morphologie des Organs insgesamt auswirken. Darüber hinaus ist eine Identifizierung von molekularen Alterungsvorgängen von großer Bedeutung, um mögliche prädiktive Faktoren für den Übergang vom physiologischen Altern in die pathologische Asthenie zu erkennen und ggf. behandeln zu können.

In der Augenheilkunde stellen insbesondere das Glaukom und die altersabhängige Makuladegeneration einen bedeutenden Anteil der visusbeeinträchtigenden Entitäten der neurodegenerativen Erkrankungen dar. Diesen beiden Erkrankungen ist gemeinsam, dass die Prävalenz für das Auftreten mit steigendem Lebensalter zunimmt. Die Ursachen der altersabhängigen Makuladegeneration sind bei isolierter Betrachtung des Auges überwiegend im vitreoretinalen Kompartiment zu identifizieren. Demgegenüber sind beim Auftreten des Glaukoms auch primäre altersbedingte Veränderungen wie des Trabekelmaschenwerkes und der Lamina cribrosa ursächlich.

In den neurowissenschaftlichen Fachgebieten werden neurodegenerative Erkrankungen wie Morbus Alzheimer als bedeutende Erkrankungen mit dem Risikofaktor Alter bewertet. Es wird davon ausgegangen, dass ca. $50 \%$ der über 85Jährigen an demenziellen Erkrankungen wie Morbus Alzheimer leiden.

Die hohe Inzidenz und Prävalenz dieser neurodegenerativen Erkrankungen führt zur großen Bedeutung dieser Krankheitsbilder sowohl in den Neurowissenschaften als auch in der Augenheilkunde. Es ergeben sich Hinweise, dass analoge Mechanismen beizerebralen und okulären neurodegenerativen Prozessen zu der Entstehung dieser Krankheiten beitragen können. Daher lässt sich vermuten, dass eine Auseinandersetzung mit klinischen, morphologischen und auch molekularbiologischen Vorgängen dieser Erkrankungen im Kontext des physiologischen Alterns zu einem besseren Verständnis neurodegenerativer Vorgänge beitragen kann. Erste klinische Erfahrungen und sich rasant entwickelnde Möglichkeiten in der ophthalmologischen Bildgebung lassen vermuten, dass okuläre Screeningverfahren bzw. die Identifikation von Biomarkern zur Diagnostik neurodegenerativer zerebraler Erkrankungen zukünftig möglich sein könnten.

Eine weitere intensive Auseinandersetzung mit dem alternden Auge und assoziierter Erkrankungen ist erforderlich, um zukünftig den Übergang von physiologischen Prozessen in krankhafte Zustände frühzeitiger zu detektieren und ggf. kausale Therapieoptionen zu ermöglichen. Es ist wünschenswert, dass durch interdisziplinäre und komparative Ansätze mit neurowissenschaftlichen Fachgebieten analoge Mechanismen detektiert sowie neue diagnostische und therapeutische Optionen abgeleitet werden können.

Halten wir es wie Leonardo da Vinci und betrachten wir auch zukünftig mögliche Verbindungen zwischen Auge und Seele. Die Beiträge zu diesem Leitthema sollen einen solchen Blick über den Tellerrand "des alternden Auges“ in das neurowissenschaftliche Fachgebiet zum zentralen Nervensystem hin ermöglichen - wohlwissend, dass diese Zeilen nur einen kleinen Bruchteil des 
aktuellen Standes der Wissenschaft abbilden können. Es gibt viel zu tun! Wir wünschen dem Leser bei der Erkundung dieses Zwischenstandes viel Vergnügen!

Ihr

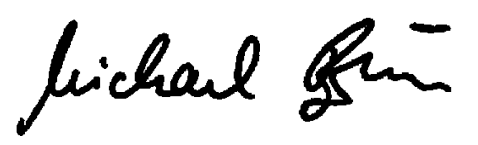

\section{Korrespondenzadresse}

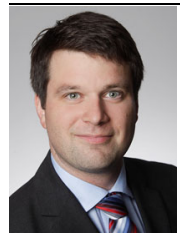

\section{R. R. Böhm}

Zentrum für Augenheilkunde, Klinik für Erkrankungen des vorderen Augenabschnittes, Universitätsklinikum Essen Hufelandstr. 55, 45147 Essen, Deutschland michael.boehm@uk-essen.de

Interessenkonflikt. M.R.R. Böhm gibt an, dass kein Interessenkonflikt besteht.

\section{Immer aktuell - Dank Online-First}

Warum warten, bis die Artikel im Heft abgedruckt sind, wenn Sie sie auch schon vorher lesen können?

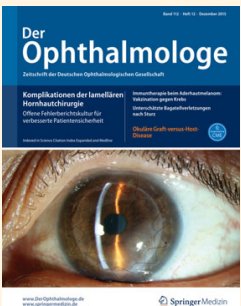

- Netzhautbuckel ohne Chirurgie. Termühlen, J., Clemens, C.R., Alten, F. et al. doi:10.1007/s00347-016-0423-5

- Patientenzufriedenheit nach ambulanter Kataraktoperation. Unterschiede zwischen ambulant in einer Praxis und ambulant im Krankenhaus operierten Patienten. Stein, I., Schoenfelder, T. \& Kugler, J. doi:10.1007/s00347-016-0422-6

- Zwei Kinder mit intermediärer Uveitis und Granuloma anulare in derselben Nachbarschaft. Nessmann, A., Klein, R., Herzer, P. et al. doi:10.1007/s00347-016-0431-5

- Intravitreales Ganciclovir als additive Therapieoption bei akuter retinaler Nekrose. Bischoff-Jung, M., Viestenz, A., Fiorentzis, M. et al. doi:10.1007/s00347-016-0398-2

- Bewertung der methodischen Qualität publizierter RCTs in der Kataraktchirurgie. Pilotstudie zur quantitativen Zielerfüllung bestehender Anforderungen laut CONSORT-Statement und deren qualitative Validierung.

Baulig, C., Krummenauer, F. \& Knippschild, S. doi:10.1007/s00347-017-0446

- Plötzliche bilaterale Visusminderung bei einem 14-jährigen Jungen. Marx, S., Scheuerle, A., Khoramnia, R. et al. doi:10.1007/s00347-017-0448-4

Übrigens: Wussten Sie schon, dass die Artikel mit Angabe der DOI (digital object identifier) und dem Zusatz „epub ahead of print" bereits zitiert werden können? 